\title{
Les évolutions récentes du droit belge dans la régulation du fait religieux (2015-2019)
}

Louis-Léon Christians et Léopold Vanbellingen

\section{OpenEdition}

Édition électronique

URL : http://journals.openedition.org/rdr/440

DOI : $10.4000 /$ rdr.440

ISSN : 2534-7462

Éditeur

Presses universitaires de Strasbourg

\section{Édition imprimée}

Date de publication : 14 novembre 2019

Pagination : 191-207

ISBN : 979-10-344-0054-6

ISSN : 2493-8637

\section{Référence électronique}

Louis-Léon Christians et Léopold Vanbellingen, « Les évolutions récentes du droit belge dans la régulation du fait religieux (2015-2019) », Revue du droit des religions [En ligne], 8| 2019, mis en ligne le 25 novembre 2019, consulté le 19 novembre 2020. URL : http://journals.openedition.org/rdr/440 DOI : https://doi.org/10.4000/rdr.440

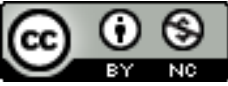

La revue du droit des religions est mise à disposition selon les termes de la Creative Commons Attribution - Pas d'Utilisation Commerciale 4.0 International - CC BY-NC 4.0. 


\section{LESÉVOLUTIONS RÉCENTESDUDROITBELGE DANSLA RÉGULATION DUFAIT RELIGIEUX (2015-2019)}

\section{Louis-Léon CHRISTIANS avec la collaboration de Léopold VANBELLINGEN}

Chaire Droit \& Religions, Université catholique de Louvain

Si les sondages semblent montrer que la société belge poursuit sa sécubien au contraire, une diminution des contentieux. Des nouveaux effets de polarisation apparaissent et le «droit» est par excellence le lieu où ces divers à-coups se manifestent. On remarquera toutefois que les causes profondes de ces «à-coups» et leur «nouveauté», demeurent ambiguës dès lors que ces polémiques rejouent souvent, au prétexte notamment de l'islam, des tensions qui étaient en fait anciennes entre catholiques et laïques.

Les polémiques bioéthiques demeurent encore essentiellement traversées par des arrière-plans conflictuels avec le catholicisme. La fluctuation des coalitions politiques conduit à des évolutions diverses voire à des compromis singuliers: par exemple, deux lois adoptées «en parallèle» en 2018, ont, pour l'une, exclu du Code pénal toutes dispositions relatives à l'avortement et transféré les limites pénales concernant l'IVG dans des lois sanitaires moins symboliques ${ }^{1}$, et, pour l'autre, permis une reconnaissance d'état civil

1. L., 15 oct. 2018 , relative à l'interruption volontaire de grossesse, abrogeant les articles 350 et 351 du Code pénal et modifiant les articles 352 et 383 du même Code et modifiant diverses dispositions législatives: M.B., 29 oct. 2019. 
au foetus mort-né, dès 140 jours au lieu de 180, pour les parents qui en font la demande ${ }^{2}$.

D'autres contentieux ont été explicitement référés à des enjeux religieux. Depuis 2015, quatre dossiers principaux ont ainsi marqué l'actualité juridique belge dans ses dimensions parlementaires tantôt fédérales, régionales ou communautaires: la réforme francophone des cours de religions/ morale à l'école publique; la prohibition de l'abattage rituel sans étourdissement dans les Régions flamande, wallonne, mais pas bruxelloise; la création d'un Institut public francophone de promotion des formations sur l'islam; les débats hésitants sur l'inscription d'un principe de laïcité dans un préambule constitutionnel (1). La période 2015-2018 a également été marquée par la menée à son terme du processus d'arbitrage Église-État destiné à résorber les cas prescrits d'abus sexuels au sein de l'Église catholique, le report de la reconnaissance publique du bouddhisme, l'instauration d'un dispositif transparent de dialogue régulier du Gouvernement avec les cultes reconnus (2). Enfin, diverses controverses et contentieux ont conduit plus ponctuellement à des décisions significatives, judiciaires ou administratives, parmi la cent-cinquantaine publiée entre janvier 2015 et janvier $2019^{3}$ (3).

\section{DES MODIFICATIONS LÉGISLATIVES}

\subsection{LA RÉFORME FRANCOPHONE DES COURS DE RELIGIONS/MORALE À L'ÉCOLE PUBLIQUE}

Le système instauré par la loi du pacte scolaire de 1959, et partiellement érigé en norme constitutionnelle en $1988^{4}$ (art. 24) prévoyait que le «cours de morale non confessionnelle» soit tenu pour subsidiaire et neutre par rapport aux cours de religions reconnues instaurés au sein de l'enseignement public, en vue de fournir un enseignement alternatif aux élèves qui ne souhaitaient pas suivre de cours de religion. Saisie par des militants laïques, la

2. L., 19 déc. 2018 , modifiant diverses dispositions relatives à la réglementation concernant l'enfant sans vie: M.B., $1^{\text {er }}$ févr. 2019.

3. V. le relevé de cette jurisprudence assuré via l'Observatoire juridique du fait religieux en Belgique, Chaire Droit \& Religions de l'Université de Louvain: http://ojurel.be [consulté le 4 juin 2019].

4. Pour un aperçu socio-historique, C. SAGESSER, Les cours de religion et de morale dans l'enseignement obligatoire, Courrier hebdomadaire du Crisp, $\mathrm{n}^{\circ}$ 2140-2141, 2012. 
Cour constitutionnelle de Belgique, dans un arrêt du 12 mars $2015^{5}$, constate a posteriori une perte de neutralité de ce cours de morale, en raison de la reconnaissance de la laïcité, en 1993, non plus comme une figure neutre, mais comme «organisation philosophique non confessionnelle» au même titre que les cultes. La Cour énonce alors que:

«(B.7.1) Dans cette situation, il ressort de la jurisprudence de la Cour européenne des droits de l'homme précitée que, pour que soit assuré le droit des parents à ce que leurs enfants ne soient pas confrontés à des conflits entre l'éducation religieuse ou morale donnée par l'école et les convictions religieuses ou philosophiques des parents, les élèves doivent pouvoir être dispensés de l'assistance au cours de religion ou de morale. (B.7.2) En outre, afin de protéger leur droit à ne pas divulguer leurs convictions religieuses ou philosophiques, qui relèvent avant tout du for intérieur de chacun (CEDH, 9 octobre 2007, Hasan et Eylem Zengin c. Turquie, § 73), la démarche à accomplir en vue d'obtenir cette dispense ne pourrait imposer aux parents de motiver leur demande de dispense et de dévoiler ainsi leurs convictions religieuses ou philosophiques (CEDH, 9 octobre 2007, Hasan et Eylem Zengin c. Turquie, § 76; 16 septembre 2014, Mansur Yalçin et autres c. Turquie, §§ 76-77).»

Le Parlement francophone va se «saisir ${ }^{6}$ de cet arrêt pour aller plus loin dans la mutation du système: non seulement pour permettre aux élèves d'être totalement dispensés des cours de religion et de «morale laique», mais aussi pour créer à leur intention un nouveau cours destiné à prendre la relève d'un enseignement réellement neutre (que l'on dénomma temporairement « cours de rien»). La réforme ne va toutefois pas s'arrêter là.

À ce cours expérimental «de rien», va succéder un cours «de philosophie et de citoyenneté ${ }^{7} »$ dont la neutralité de contenu semblerait garantie

5. L.-L. Christians, M. El Berhoumi, «De la neutralité perdue à l'exemption du cours de morale», Journal des tribunaux 2015, p. 437-447; X. Delgrange, A. Overbeeke, «IV.2. - Le choix entre l'enseignement d'une des religions reconnues et celui de la morale non confessionnelle» in Les Grands arrêts en droit de l'enseignement, Bruxelles, Larcier, 2016, p. 242-280.

6. R. BRAEKEN, «Cours dits philosophiques (religions et morale non confessionnelle) et cours de philosophie et de citoyenneté - Un jeu de casquettes idéologiques? », Scolanews 2016, liv. 4, p. 7-9; X. Delgrange, «Le sort du cours de morale: activisme juridictionnel contre attentisme politique», Administration publique trimestrielle, 2015/2, p. 241-265.

7. D., 22 oct. 2015, relatif à l'organisation d'un cours et d'une éducation à la philosophie et à la citoyenneté: M.B., 9 déc. 2015; D., 13 juill. 2016, relatif à la mise en ouvre d'un cours de philosophie et de citoyenneté dans l'enseignement fondamental ainsi qu'au maintien de l'encadrement pédagogique alternatif dans l'enseignement secondaire: M.B., 10 août 2016; D., 19 juill. 2017 relatif à la mise en œuvre d'un cours de philosophie et de citoyenneté 
de façon plus ferme et plus pérenne. Ce cours ne sera plus seulement un cours alternatif pour les élèves dispensés, mais devient un cours obligatoire pour tous les élèves.

Ce nouveau cours obligatoire pourrait-il à terme se substituer totalement aux cours de religions/morale? Certains partis l'ont encore souhaité dans leurs programmes électoraux de 2019. Mais le nouveau cours, n'étant pas doté d'un statut constitutionnel, à la différence des cours de religions/morale, n'a finalement obtenu du législateur francophone qu'une substitution partielle. Les cours de religions/morale, initialement de deux heures par semaine, sont réduits à une période d'une heure. L'autre heure étant affectée au nouveau cours de philosophie et citoyenneté, pour tous.

Enfin, la question des titulaires de ce nouveau cours s'est posée. Face à des pressions syndicales s'opposant à toute perte d'emploi, ce sont les professeurs de morale laïque (désormais non neutre), mais aussi ceux des religions reconnues qui ont été admis, moyennant conditions, à donner l'heure nouvelle de citoyenneté, en vue de conserver leur horaire initial. Une formation nouvelle «à la neutralité» a été organisée pour obtenir cette habilitation. Une fois désignés pour les deux cours, les enseignants sont considérés comme non neutres dans la part horaire «ancienne» (de l'heure de religions/morale) et déontologiquement neutres pour la part horaire nouvelle (du cours de citoyenneté). Invoquant la nécessité d'éviter toute dérive émotionnelle des élèves, le législateur a prévu qu'un même enseignant ne puisse pas assurer les deux fonctions au sein d'une même implantation scolaire. On comprendra aisément que les contentieux ne manquent pas à chaque stade de ces mutations profondes ${ }^{8}$.

À ce cadre d'extinction, succédera plus tard un cadre d'enseignants titulaires de diplômes spécifiques, notamment d'un master en philosophie.

Tout ceci ne concerne toutefois que la partie francophone de la Belgique. Le système antérieur, dit du pacte scolaire de 1959, subsiste en Régions flamande et germanophone.

dans l'enseignement secondaire et portant diverses adaptations dans l'enseignement fondamental: M.B., $1^{\text {er }}$ sept. 2019. V. X. Delgrange, «La Belgique francophone accouche douloureusement d'un cours de philosophie et de citoyenneté non désiré par tous », Revue $\mathrm{du}$ droit des religions, $\mathrm{n}^{\circ}$ 5, 2018, p. 107-132.

8. V. notamment deux arrêts de la Cour constitutionnelle, n 39/2018 du 29 mars 2018 et $n^{\circ}$ 114/2018 du 19 juillet 2018, et X. Delgrange, «Cours de philosophie et de citoyenneté, la cour constitutionnelle adepte du stoïcisme philosophique et de l'égalité citoyenne», Administration publique trimestrielle, 2018/3, p. 256-271. 


\subsection{LA PROHIBITION DE L'ABATTAGE RITUEL SANS ÉTOURDISSEMENT DANS LES RÉGIONS FLAMANDE ET WALLONNE (MAIS NON EN RÉGION BRUXELLOISE)}

Face aux débats nouveaux sur le bien-être animal, liés notamment à la régionalisation de cette matière, le Conseil d'État de Belgique, en sa section de législation, a toujours estimé qu'une interdiction absolue de l'abattage sans étourdissement contreviendrait de manière disproportionnée à la liberté de religion des fidèles concernés ${ }^{9}$. Selon le Conseil d'État, une prohibition «rendrait trop difficile, pour un certain nombre de croyants, la possibilité d'acheter et de consommer de la viande réputée conforme à leurs préceptes religieux». Par ailleurs, dans ces mêmes avis, le Conseil d'État s'aventure sur le terrain - glissant? - de la comparaison entre droits de l'homme et «droits des animaux» en précisant que, si «le bien-être animal doit être reconnu et que cette cause peut justifier certaines restrictions de la liberté de religion», "l'appréciation de la limitation d'un droit fondamental sur base du bien-être animal n'est pas similaire à l'appréciation de la limitation d'un droit fondamental au profit d'un autre droit fondamental». Le Conseil d'État clôt ses avis en suggérant au législateur de rechercher des «méthodes alternatives $[\ldots]$ - par exemple via les méthodes d'abattage et le contrôle de celles-ci - permettant de réduire la souffrance animale sans méconnaître la liberté de religion», «en dialogue» avec les parties concernées.

À la différence du Parlement de la Région de Bruxelles, les législateurs tant flamand ${ }^{10}$ que wallon ${ }^{11}$ ont mis un terme aux pratiques accommodantes antérieures ${ }^{12}$. Ils ont adopté en 2017 deux décrets rendant absolument obligatoire un étourdissement préalable de l'animal, sans plus donner suite à la dérogation prévue pour les abattages rituels par le règlement de l'Union européenne. Les deux décrets prévoient toutefois un procédé d'étourdissement alternatif pour l'abattage effectué dans le cadre d'un rite religieux, fondé sur l'étourdissement réversible et sur le précepte selon lequel l'étourdissement ne

9. Section de législation du Conseil d'État, avis n 60.870 et 60.871, 20 févr. 2017: Doc. parl., Parlement wallon, 604/2 (2016-2017); avis $\mathrm{n}^{\circ} 59.484 / 3$ et 59.485/3, 29 juin 2016: Doc. parl., Parlement flamand, 2014-2015, $\mathrm{n}^{\circ} 111 / 2$ et $\mathrm{n}^{\circ} 351 / 2$; avis $\mathrm{n}^{\circ} 40.350 / \mathrm{AG}$, 21 juin 2006: Doc. parl., Sénat, 2005-2006, n³ 3-808/6.

10. D. de la Région flamande, 7 juill. 2017, portant modification de la loi du 14 août 1986 relative à la protection et au bien-être des animaux: M.B., 18 juill. 2017.

11. D. de la Région wallonne, 18 mai 2017, modifiant les articles 3, 15 et 16 et insérant un article 45ter dans la loi du 14 août 1986 relative à la protection et au bien-être des animaux: M.B., $1^{\mathrm{er}}$ juin 2017.

12. V. sur l'historique de ce contentieux en Belgique, C. SAGESSER, Les débats autour de l'interdiction de l'abattage rituel, Courrier hebdomadaire du Crisp, n 2385, 2018. 
peut entraîner la mort de l'animal. Cette clause, jugée sans pertinence par les communautés juives de Belgique, semble avoir été négociée au regard de certains courants moins stricts de l'islam, dont les usages religieux ne rejetteraient que les étourdissements irréversibles.

Dès lors que la Cour de Justice de l'Union européenne (CJUE) ${ }^{13}$ semble avoir indiqué que des abattoirs "temporaires» ne pouvaient rencontrer les exigences de l'Union, les difficultés redoublent à l'occasion des jours de fêtes religieuses et en particulier pour les communautés nombreuses. Deux recours, d'associations juives et musulmanes, ont dès lors été introduits devant la Cour constitutionnelle de Belgique pour violation de la liberté de religion par ces décrets. Si l'un n’a pas abouti pour des raisons de procédure ( $\left.\mathrm{n}^{\circ} 52\right)$, l'autre a conduit la Cour constitutionnelle de Belgique ${ }^{14}$ à poser une question préjudicielle à la Cour de Justice de l'Union sur la conformité de ces décrets aux droits fondamentaux de l'Union et en particulier quant à leur caractère éventuellement discriminatoire au regard du fait que les conditions posées à l'abattage religieux ne le sont pas «dans le cadre de la chasse, de la pêche et de manifestations culturelles et sportives ».

\subsection{LA CRÉATION D'UN INSTITUT PUBLIC FRANCOPHONE DE PROMOTION DES FORMATIONS SUR L'ISLAM (IPFI)}

La formation des responsables musulmans, mais aussi plus généralement la formation à l'islam, fait l'objet d'une attention croissante des autorités belges. La perspective très tôt apparue est de ne pas totalement séparer un apport de sciences humaines, à charge des universités, et un apport théologique, qui serait à la seule charge des autorités religieuses. C'est une interaction qu'il s'agit de promouvoir, tout en assumant une exigence de neutralité de l'État et de respect mutuel entre autorités publiques et religieuses. À l'issue des travaux d'une Commission d'experts ${ }^{15}$ mandatée par le ministre de l'Enseignement supérieur, et après des avis très vigilants du Conseil d'État ${ }^{16}$, le

13. CJUE, Gde ch., 29 mai 2018, C-426/16, Liga van Moskeeën en Islamitische Organisaties Provincie Antwerpen VZW e. a., V. S. WATTIER, «Arrêt "Liga van Moskeeën en Islamitische Organisaties": l'obligation d'effectuer l'abattage rituel dans un abattoir agréé au regard du droit à la liberté de religion», Journal du droit européen, 2018/10, p. 385-387.

14. Cour constitutionnelle de Belgique, 4 avril 2019, nos 52 et 53/2019.

15. A. ReA et F. Tulkens, Rapport de la Commission concernant la formation des cadres musulmans et les émissions concédées, 2015: https://cdn.uclouvain.be/public/Exports\%20reddot/ cismoc/documents/Rapport_final_commission_Marcourt(1).pdf [consulté le 4 juin 2019].

16. CE, 15 juin 2016, avis 59.411 et 29 août 2016, avis 59.996: http://www.raadvst-consetat. be [consulté le 4 juin 2019]. 
législateur francophone a créé par décret du 16 décembre $2016^{17}$ un institut de droit public non pas de «formation en matière d'islam», dont l'organisation ne peut relever des compétences d'un État neutre, mais bien d'un institut «de promotion des formations sur l'islam» qui a pour mission de:

«1. identifier les formations existantes sur l'islam en Communauté française et plus largement en Belgique; 2. proposer, soutenir et financer des formations à destination des imams, des maîtres et professeurs de religion islamique, des conseillers islamiques, des acteurs socioculturels ou tout autre public intéressé par l'islam; 3. assurer la mise en réseau de ces formations; 4. organiser des conférences et des conférencesdébats; 5 . travailler à la création d'un "bachelier" (selon le lexique belge) en sciences religieuses et sociales et d'un master en théologie musulmane; 6 . préparer les modifications à apporter aux dispositions législatives et réglementaires en vigueur; 7 . préparer les accords de coopération nécessaires; 8 . travailler à la création, soutenir et financer une "Chaire interuniversitaire d'islamologie pratique" annuelle visant l'analyse réflexive, critique, de la pensée arabo-musulmane dans ses dimensions historiques et contemporaines. Il appartient aux seules institutions d'enseignement de décider si les compétences dispensées dans le cadre de cette chaire interuniversitaire sont obligatoires, à quels types de publics elles se destinent, ainsi que leurs modalités d'évaluation; 9. poursuivre la réflexion au sujet de la création d'une "Faculté de théologie musulmane", en collaboration avec la Communauté flamande si possible.»

\subsection{LES DÉBATS HÉSITANTS SUR L'INSCRIPTION D'UN PRINCIPE DE LAÏCITÉ DANS UN PRÉAMBULE CONSTITUTIONNEL}

En avril 2019, au moment de fixer les limites d'une future révision de la Constitution belge pour 2020-2025, aucun parti en commission, à l'exception des socialistes et des libéraux flamands, n'a voté en faveur de la révision du titre II de la Constitution («Des Belges et de leurs droits») et de l'insertion d'un article relatif à la laïcité de l'État, ce qui aurait permis de prolonger les débats entamés au Parlement, qui avaient notamment donné lieu à de vastes auditions d'experts désignés par les divers partis ${ }^{18}$. On verra probablement

17. M.B., 25 janv. 2017.

18. Rapport introductif d'initiative parlementaire, Le caractère de l'État et les valeurs fondamentales de la société, Doc. parl. Chambre, 54-2914/001 (24 janv. 2018). En dehors du rapport, V. par ex. les analyses de H. HASQUIN, Inscrire la laïcité dans la Constitution belge?, Éd. de l'Académie Royale de Belgique, 2016; V. DE CoOrebyter, "Comment conclure le débat constitutionnel sur la laïcité?», Le Soir, 17 oct. 2018. 
dans cet enlisement une nouvelle conséquence de l'ambiguité juridique du concept belge de laïcité, qui désigne à l'heure actuelle une organisation philosophique non confessionnelle, reconnue et financée par l'État au même titre que les cultes reconnus, et qui est frappée, comme eux jusqu'à présent, d'un principe de séparation.

\section{QUELQUES DISPOSITIFS EN COURS}

\subsection{LA FIN DU PROCESSUS D'ARBITRAGE ÉGLISE-ÉTAT DESTINÉ À RÉSORBER LES CAS PRESCRITS D'ABUS SEXUELS AU SEIN DE L'ÉGLISE CATHOLIQUE}

Le 6 mars 2017, le rapport final du Comité scientifique du Centre d'arbitrage en matière d'abus sexuels a été déposé au Parlement belge ${ }^{19}$. Ce rapport de 312 pages est le dernier acte d'un processus majeur de coopération entre le Parlement et l'Église catholique pour reconnaître et indemniser l'ensemble des victimes déclarées d'abus sexuel dans des relations pastorales, mais dont les prescriptions pénales et civiles empêchaient qu'elles soient encore entendues en droit belge ou en droit canonique.

On rappelle brièvement que la Conférence des évêques de Belgique avait décidé unilatéralement, en 1998, de créer une commission consultative pour le suivi de toute plainte canonique. De 1999 à 2010, cette commission, dite Halsberghe du nom de sa présidente, magistrate retraitée, a reçu et traité une trentaine de dossiers prescrits pénalement, signalés par des victimes via un « $\mathrm{n}^{\circ}$ de téléphone vert». En 2010, au moment où une nouvelle commission ecclésiale se mettait en place, dite Adriaenssens, du nom de son président-psychiatre, professeur à l'Université de Leuven, un scandale ébranla la Flandre: l'inculpation de l'évêque de Bruges pour abus anciens sur un de ses neveux. Près de cinq cents signalements affluèrent alors en quelques jours auprès de la nouvelle commission, qui entra en contact avec le Parquet général de Belgique pour trouver un modus vivendi. L'ensemble des dossiers furent saisis quelques jours plus tard, mettant de ce fait un terme aux travaux de cette commission ecclésiale.

Le Parlement belge crée alors en son sein, en octobre 2010 une « Commission spéciale relative au traitement d'abus sexuels et de faits de pédophilie dans une relation d'autorité, en particulier au sein de l'Église», qui a entendu

19. Rapport final du Comité scientifique du Centre d'arbitrage en matière d'abus sexuels, Doc. parl. Chambre, 54-767/004 (6 mars 2017). 
l'ensemble des acteurs. Dans son rapport du 31 mars 2011, cette commission, malgré beaucoup d'émoi, ne suggéra finalement pas de rendre les abus sur mineurs imprescriptibles, ni en droit d'État, ni en droit canonique. Se saisissant du poids moral de ces affaires, la commission obtient en revanche un accord global avec l'Église catholique pour créer un processus mixte et transparent dit «d'arbitrage» par lequel toutes les victimes d'affaires prescrites qui s'y déclareraient avant le 31 octobre 2012 verraient leur cas examiné de façon objective, et le cas échéant reconnu et indemnisé de façon forfaitaire, sans pouvoir en revanche décider aucune imputation pénale à charge des auteurs présumés. Le coût de fonctionnement de ce dispositif a été partagé entre l'Église pour $67 \%$ et l'État pour $33 \%$. Des indemnités ont été octroyées pour un montant global d'environ 3 millions d'euros (à charge de l'Église catholique), couvrant 600 dossiers, dont 500 avec demande d'indemnisation. En 2019, la Conférence des évêques a, à son tour, publié un rapport global couvrant l'ensemble de constats et des actions de l'Église catholique en Belgique ${ }^{20}$.

\subsection{LE REPORT DU STATUT DE «PHILOSOPHIE RECONNUE » POUR LE BOUDDHISME}

Après plusieurs années de négociation ${ }^{21}$, une loi du 24 juillet 2008 portant des dispositions diverses (art. 139) 22 octroie aux représentants des communautés bouddhistes une subvention fédérale temporaire, en vue de soutenir leur préparation d'une future reconnaissance légale du bouddhisme comme culte reconnu. En avril 2019, une proposition de loi a été déposée au Parlement en vue de cette reconnaissance, sans être adoptée en raison de la fin de la mandature ${ }^{23}$. Dix ans se sont donc à tout le moins écoulés depuis

20. ÉVÊQues et SuPÉRIeURS De Belgique, Abus sexuels de mineurs dans une relation pastorale dans l'Église de Belgique. Vers une politique cohérente, 1995-2017, Bruxelles, 12 févr. 2019: https://www.kerknet.be/sites/default/files/19\%2002\%2012\%20Rapport\%20Abus\%20 sexuels\%20de\%20mineurs.pdf [consulté le 4 juin 2019].

21. B. DE BACKER, Bouddhismes en Belgique, Courrier hebdomadaire du CRISP, n 1768-1769, 2002.

22. V. A. royal, 20 nov. 2008, portant réglementation relative à l'octroi de subsides à l'association sans but lucratif « Union bouddhique belge» - «Boeddhistische Unie van België»: M.B., 5 déc. 2008.

23. Proposition de loi relative à l'Union bouddhique belge, aux délégués et aux établissements chargés de la gestion des intérêts matériels et financiers des communautés bouddhiques reconnues, Doc. parl. Chambre, 54-3705 (2 avr. 2019). V. également, Proposition de loi relative au subventionnement du Forum Hindou de Belgique, Doc. parl. Chambre, 54-3704 (2 avr. 2019). 
l'amorce d'un processus de reconnaissance ${ }^{24}$. Cette lenteur est imputée par les commentateurs d'une part à la priorité donnée à l'accroissement du financement du culte islamique (notamment pour une augmentation des postes de conseillers islamiques dans les prisons) et d'autre part au fait que le bouddhisme belge entend être reconnu non pas comme religion, mais comme philosophie, au même titre que la laïcité organisée belge. Si un tel choix est effectivement libre, et s'il conduit à une pleine compétence de l'État fédéral pour la réglementation des communautés locales (à la différence de la réglementation des cultes, partiellement régionalisée en 2001), il n'en reste pas moins que les cours de religions et de morale ont été organisés à l'école publique, en Flandre comme en Fédération Wallonie-Bruxelles, en conférant plus ou moins implicitement le cours de morale à la seule laïcité organisée. Insérer le bouddhisme en alter ego de la morale laique reste un défi singulier.

\subsection{UN DISPOSITIF TRANSPARENT DE DIALOGUE RÉGULIER DU GOUVERNEMENT AVEC LES CULTES RECONNUS}

Après de premières rencontres informelles en 2015 et 2016 avec les représentants des cultes et philosophies reconnus, le Premier ministre Charles Michel (MR) et le ministre de la Justice Koen Geens (CD\&V) ont cosigné, le 17 mai 2017, avec les représentants des cultes reconnus et de la laïcité, un protocole d'accord visant à instituer un dialogue entre l'autorité fédérale et les organes des cultes et de la laïcité organisée. À cette fin, un «Conseil a été créé; il se réunira au moins deux fois par an sous la houlette du Premier ministre et du ministre de la Justice, mais également autant de fois que nécessaire, voire en urgence, si les circonstances l'exigent».

Un dialogue spécifique avec les cultes et philosophies reconnus avait déjà été inauguré par le Gouvernement flamand en 2014 (Vlaamse Interlevensbeschouwelijke Dialoog - VLID), puis institutionnalisé en une plateforme de concertation formelle. En 2017, cette plateforme a signé une charte qui affirme notamment que «malgré leurs différences profondes, les représentants des cultes et philosophies reconnus se retrouvent dans un objectif commun: un monde vivable, une société du bien-être pour tous, la liberté, la paix, la

24. A. Overbeeke, «Belgisch boeddhisme, nog steeds niet-erkend: het eredienstenrecht op dood spoor? », Actua Leges, 2019/2; «La (non-) reconnaissance du bouddhisme - indice d'un droit des cultes au point mort?", Commentaires de la Chaire de droit des religions, 3 janv. 2019: http://ojurel.be [consulté le 4 juin 2019]. 
tolérance et le maintien de l'État de droit démocratique». Les autres Régions et Communautés n'ont pas encore formalisé de tels dispositifs ${ }^{25}$.

\section{PARMI LES CONTENTIEUX ET JURISPRUDENCES}

Parmi la cent-cinquantaine de décisions publiées entre janvier 2015 et janvier 2019, les contentieux les plus fréquents concernent l'islam, mais d'autres demeurent significatifs à propos du catholicisme ou de la laicité militante. Parmi les thèmes les plus récurrents, outre le bien-être animal et l'abattage rituel déjà évoqués, on trouve l'asile pour cause religieuse (avec des controverses relatives à certaines dérives imputées à l'accueil de chrétiens de Syrie, mais aussi concernant un nombre particulier de victimes de pratiques vaudou), le statut des religions dans l'enseignement public (signes religieux, statut du cours de morale, liberté d'expression des professeurs de religions ${ }^{26}$ ), le statut des détenus (généralement musulmans ${ }^{27}$ ), le temporel des cultes reconnus (généralement des fabriques d'église catholique et de leur tutelle administrative), et les contentieux les plus variés concernant le droit de la non-discrimination ${ }^{28}$. Quelques rares décisions publiées concernent la religion en droit de la famille et droit international privé. Une seule décision publiée concerne la laïcité organisée (à propos de la reprise en main par son

25. P. De Pooter, L.-L. Christians (eds), Les dispositifs publics de dialogue interconvictionnel, Bruxelles, Larcier, à paraître; A. OvERBEEKE, «De Staat en de vele geloofsgemeenschappen: raadgever-coach? Regisseur? Regelaar? De fragiele positie van de overheid als 'neutrale organisator van religie-uitoefening' », Recht, Religie, Samenleving, 2016/1, p. 19-68.

26. L.-L. Christians, X. Delgrange, « IV.3. - Le contrôle du respect des droits fondamentaux dans les enseignements dispensés " in Les Grands arrêts en droit de l'enseignement, Bruxelles, Larcier, 2016, p. 281-301.

27. Par ex., CE, 24 janv. 2019, n 243.480, Benameur.

28. Dont une jurisprudence de plus en plus exigeante quant aux conditions de refus du port de signes religieux, en dehors du cas des agents publics au sens strict. V. par ex. les commentaires belges de l'arrêt Lachiri de la Cour EDH, 18 déc. 2018, condamnant la Belgique à propos de la prohibition d'un foulard porté par une partie civile dans une salle d'audience: M. CADELLI, «Interdiction du voile à l'audience: quand le juge est l'ennemi», Journal des tribunaux, 2019/13, p. 253-260; D. Koussens et X. Delgrange, «Quelles laïcités en salle d'audience? À propos de quelques arrêts canadiens et européens sur le port de symboles religieux dans les prétoires», RTDH 2019, p. 447-475 et s. OuAlD-CHAib, «Hamidovic c. Bosnie-Herzégovine: l'interdiction de couvre-chef religieux dans les prétoires viole l'article 9 de la Convention européenne des droits de l'homme», Journal des tribunaux, 2018/19, p. 401-405; V. aussi à propos de stagiaires dans le cadre des services d'aide à l'emploi: I. RORIVE, «Être et avoir l'air: une scénographie baroque des principes de neutralité et de non-discrimination. Commentaire de l'ordonnance du Tribunal du travail francophone de Bruxelles siégeant comme en référé du 16 novembre 2015 », Administration publique trimestrielle, 2016/4, p. 491-516. 
organe représentatif d'une émission concédée de la télévision publique ${ }^{29}$ ). Enfin, on doit souligner l'importance croissante des prises de position de divers organes publics, comme le Centre interfédéral pour la lutte contre la discrimination (UNIA), le Comité national de bioéthique ou encore les ordres professionnels.

Dans les lignes qui suivent, on évoquera le licenciement de salariées d'entreprises privées en raison du port du voile, le refus du burkini dans certaines piscines publiques, l'opposition à célébrer le mariage civil lorsque la future épouse refuse de serrer la main de l'officier d'état-civil ; les positions du Comité national belge de bioéthique sur la circoncision; les positions du Conseil de l'Ordre des médecins relatives à la pratique des certificats de virginité.

\section{1. «ENTREPRISE NEUTRE » ET PORT DU FOULARD ISLAMIQUE PAR DES SALARIÉES}

Le recours à une règle de neutralité comme outil de gestion du fait religieux dans l'entreprise privée devient un thème majeur en droit de la non-discrimination. Les arrêts rendus en mars 2017 par la CJUE dans les affaires Achbita c. G4S et Bougnaoui c. Micropole ont suscité de multiples interrogations quant à la manière dont les juridictions nationales seraient amenées à intégrer les attendus de Luxembourg dans leurs jurisprudences respectives ${ }^{30}$.

Une décision rendue un an plus tard en Belgique fournit une première indication à ce sujet. Dans un jugement inédit du 28 mai $2018^{31}$, le tribunal du travail francophone de Bruxelles est confronté à une affaire dont les faits litigieux entrent en résonnance directe - ce que reconnaît d'ailleurs le tribunal - avec ceux au cœur de l'arrêt Achbita. Ce jugement concerne le licenciement d'une employée vendeuse au sein de l'enseigne d'électroménager

29. CE, 5 sept. $2014, \mathrm{n}^{\circ} 228.301$ et 14 avr. 2016, n 234.401, commentés par F. JONGEN, A. Strowel, «Chapitre 4. - Obligations de programmation» in Droit des médias et de la communication, Bruxelles, Larcier, 2017, p. 591-601.

30. L.-L. Christians, L. VAnbellingen, « Neutralités d'entreprise et neutralités d'État: tendances asymétriques en droit belge», Droit social, 2018, p. 337-343; F. DORSSEMONT, "Liberté de religion sur le lieu de travail et la Cour de Justice: de retour à cuius regio, illius religio?» [en néerlandais], Recht, Religie, Samenleving, 2016/2, p. 65-105. ; F. KéFER et R. LinGUELET, «L'affaire Achbita c. G4S Secure Solutions: le suspense continue», Jurisprudence de Liège, Mons, Bruxelles, 2018/3, p. 122-129.

31. Trib. trav. Bxl (fr.), 28 mai 2018, R.G. no 16/7231/A, inéd: https://www.unia.be/fr/ jurisprudence-alternatives/jurisprudence/tribunal-du-travail-bruxelles-28-mai-2018 [consulté le 4 juin 2019]. 
Vanden Borre, à la suite de son intention répétée de porter un foulard islamique sur son lieu de travail, malgré l'interdiction de son employeur. Cette interdiction était fondée, comme dans l'affaire Achbita, sur une règle non écrite - réputée implicite - de neutralité, qui implique l'interdiction d'afficher des signes politiques, philosophiques ou religieux dans le chef des travailleurs. Le tribunal reconnaît la légitimité du principe de neutralité et de l'interdiction qui en découle. Il conclut en premier lieu à l'absence de discrimination directe, comme l'y invite la CJUE, du fait du caractère général de l'interdiction. L'existence d'une discrimination indirecte est tout autant rejetée, dès lors que, aux yeux du tribunal, l'interdiction a été appliquée de manière «cohérente et systématique» (CJUE, Achbita, § 40). Le fait que l'interdiction soit indistinctement applicable au personnel entrant ou non en contact avec la clientèle ne semble pas modifier les conclusions du tribunal, alors que la CJUE insistait précisément sur cette distinction pour évaluer le caractère «strictement nécessaire» de la mesure (Achbita, § 42).

Quant à la possibilité d'un changement de poste vers une fonction en arrière-boutique, sans contact avec la clientèle, suggérée également par la CJUE (Achbita, § 43) comme critère à prendre en compte dans le test de proportionnalité, et sur laquelle insistait UNIA - Centre interfédéral pour l'égalité des chances - en tant que tiers intervenant, le tribunal considère que preuve a été faite par Vanden Borre d'une impossibilité de reclassement en l'espèce.

À la différence de l'affaire Achbita, le licenciement de la présente employée était fondé sur un motif grave, n'ayant donné lieu à aucune indemnité compensatoire. Le tribunal considère qu'un tel motif grave n'est pas avéré, et accorde à l'employée une indemnité de rupture de contrat.

Cette première jurisprudence belge «post-Achbita» s'inscrit dans la droite ligne de ce que d'aucuns craignaient comme une «porte entrouverte»par la cour de Luxembourg quant à la justification systématique d'interdictions des signes religieux dans l'entreprise par l'invocation idéologique d'un principe de neutralité de droit privé.

\subsection{LE REFUS DU BURKINI DANS CERTAINES PISCINES PUBLIQUES}

À la suite des polémiques françaises de 2016, le port du burkini a suscité également quelques controverses en Belgique, non sur les froides plages de la mer du Nord, mais bien dans certaines piscines publiques. UNIA, l'autorité anti-discrimination belge, confirme que même en piscine publique une telle 
interdiction doit être considérée comme discriminatoire. Dans un avis formel $\mathrm{n}^{\circ} 166 \mathrm{du} 10$ juillet $2017^{32}$, UNIA estime qu'« un pouvoir public qui voudrait la restreindre doit avancer de solides arguments tels que la protection de l'intérêt général, la protection des droits d'autrui, etc. UNIA a dès lors soumis les arguments invoqués à diverses instances spécialisées (en genre, en hygiène, etc.). Elles estiment que les arguments évoqués (hygiène, sécurité, égalité homme/femme, réactions négatives d'autres nageurs) ne peuvent pas justifier une interdiction du maillot de bain couvrant le corps». UNIA conclut qu'une interdiction générale du port du maillot de bain couvrant le corps risquerait d'être discriminante pour «certains nageurs, notamment qui veulent le porter par conviction ou par nécessité, en raison notamment de leur état santé, d'une caractéristique physique ou d'un handicap».

Une décision du tribunal de première instance de Gand du 5 juillet $2018^{33}$ interroge la Cour constitutionnelle quant à la notion de discrimination indirecte que ne constituerait pas à ses yeux la prohibition du burkini en piscine. Une décision du tribunal de première instance d'Anvers, du 18 décembre 2018, frappée d'appel, décide en sens contraire que l'interdiction du burkini est immédiatement justifiée par des raisons de sécurité et d'hygiène ${ }^{34}$.

\subsection{L'OPPOSITION À CÉLÉBRER UN MARIAGE CIVIL LORSQUE LA FUTURE ÉPOUSE REFUSE DE SERRER LA MAIN DE L'OFFICIER D'ÉTAT-CIVIL}

Le refus de serrer la main, pour motifs religieux, a soulevé en Belgique plusieurs contentieux d'emploi fort médiatisés ${ }^{35}$. Une nouvelle forme de polémique est toutefois apparue. Plusieurs officiers d'état-civil se sont en

32. https://www.unia.be/fr/legislation-et-recommandations/recommandations-dunia/avis-uniarepond-a-des-questions-sur-le-maillot-de-bain-couvrant-le-corps [consulté le 4 juin 2019].

33. Civ. Gand, 5 juill. 2018: Tijdschrift voor Jeugd en Kinderrechten, 2019/1, p. 110-115.

34. Civ. Anvers, 18 déc. 2018: Tijdschrift voor Jeugd en Kinderrechten, 2019/1, p. 116-125, note M. SPINOY, «L'interdiction du burkini: les eaux troubles du droit de la discrimination» [en néerlandais].

35. Par ex., CE, 21 févr. 2019, n 243.770 (à propos de la non-promotion d'un agent de propreté de la Ville de Bruxelles); la presse évoque plusieurs cas de sanctions disciplinaires ou de révocation, mais aucune évaluation de la fréquence de tels contentieux n'est possible: "Refus de serrer la main à son échevine: cinq jours de retenue sur salaire», RTBF, 17 août 2013: https://www.rtbf.be/info/societe/detail_refus-de-serrer-la-main-ason-echevine-cinq-jours-de-retenue-sur-salaire?id=8068864; «Un employé de la Ville de Bruxelles, converti à l'islam, refuse de serrer la main de Karine Lalieux "à cause de sa religion" : il a été viré...», Sudinfo.be, 13 mai 2013: http://www.sudinfo.be/722264/article/ regions/bruxelles/actualite/2013-05-12/un-employe-de-la-ville-de-bruxelles-converti-al-islam-refuse-de-serrer-la [consultés le 4 juin 2019]. 
effet opposés à célébrer le mariage de couples musulmans dont l'un des membres refusait de leur serrer la main. Ainsi, en mai $2018^{36}$, l'échevin de la ville de Malines justifiait son refus en indiquant que la future épouse «a délibérément refusé de me serrer la main quand j'ai voulu la saluer, elle et son mari. Elle s'est référée à sa religion et à ses coutumes, écrit-il. Je lui ai fait comprendre qu'une telle salutation faisait partie de notre culture et de nos habitudes, certainement lors d'un mariage, que c'était une marque de respect. Les raisons religieuses n'ont pas leur place à l'hôtel de ville», poursuit-il.

Le Centre interfédéral de lutte contre la discrimination (UNIA) a pris une position publique (intitulée «carte blanche»), dès le 15 décembre 2016, pour contester de tels refus, dès lors qu'ils ne trouvent aucun fondement dans la loi belge. Dès lors que les conditions légales fixant la validité du mariage sont réunies, il n'appartient pas à un officier d'état-civil d'imposer un rituel complémentaire, ni au titre des usages, ni à celui de la neutralité. Pour UNIA, c'est tout au contraire l'officier d'état-civil qui, par son refus, «demande en fait de ne pas accomplir un acte qui relève de ses fonctions, de ne pas se soumettre à des prescrits légaux, au nom de ses convictions. Ce qu'il demande en fait, c'est de pouvoir bénéficier d'un accommodement raisonnable en raison de ses convictions. Si l'on suit ce raisonnement, il sera alors possible pour un fonctionnaire de refuser de marier un couple homosexuel $^{37}$ ».

\subsection{LES POSITIONS DU COMITÉ NATIONAL BELGE DE BIOÉTHIQUE SUR LA CIRCONCISION}

La circoncision masculine, à la différence de l'excision féminine ${ }^{38}$, n’a fait l'objet jusqu'à présent d'aucune législation en Belgique. Comme rite, cette

36. «Elle dédaigne serrer la main de l'échevin, résultat: mariage refusé», La libre Belgique, 8 mai 2018: https://www.lalibre.be/actu/belgique/elle-dedaigne-serrer-la-main-de-lechevin-resultat-mariage-refuse-5af07ef9cd7028f079d5e908 [consulté le 4 juin 2019].

37. UNIA, «Carte blanche: "À chacun sa loi ?" », 15 déc. 2016: https://www.unia.be/fr/articles/ carte-blanche-a-chacun-sa-loi [consulté le 4 juin 2019]. V. L. VAnBELLingEN, "Pour se marier, demain la main», Commentaires de la Chaire de droit des religions de l'UCLouvain: http://ojurel.be/2018/05/08/demander-la-main-se-marier/ [consulté le 4 juin 2019].

38. V. L.-L. ChRISTIANS, « La circoncision rituelle face aux droits contemporains », in R. BURNET et D. Luciani, La circoncision aujourd'hui, Paris, Éd. Feuilles, 2014, p. 69-89; X. DelGRANGE, H. LEROUXEL, "La circoncision rituelle en Europe: vers une tension entre la liberté de religion des parents et l'intégrité physique de l'enfant?», in D. Koussens et al. (dir.), La religion hors la loi, Bruxelles, Bruylant, 2016, p. 155-186; L.-L. Christians, X. Delgrange, H. Lerouxel, «La circoncision rituelle en droit belge», in V. Fortier (dir.), La circoncision rituelle, enjeux de droit, enjeux de vérité, Strasbourg, Presses universitaires de Strasbourg, 2016, p. 160-176. 
intervention fréquente n'en est pas pour autant formellement privilégiée ni immunisée de toute investigation pénale. Elle relève simplement, comme toute réalité sociale, de l'application du droit commun qu'il s'agisse du droit de la responsabilité civile, du droit pénal en matière d'exercice illégal de la médecine, ou de protection de l'intégrité physique, du régime de l'autorité parentale en droit civil, ou encore des droits de l'enfant et en l'occurrence de la liberté de religion.

Le Comité national belge de bioéthique a été saisi de ces questions en 2014. La procédure s'est conclue par un avis important rendu en 2017 qui porte « uniquement sur les situations où la circoncision est réalisée en dehors de tout contexte thérapeutique médical, soit par un médecin, soit par une autre personne ${ }^{39} »$. Quatre questions ont été reformulées par le comité: Est-il éthiquement admissible de procéder à une circoncision en dehors de toute indication médicale? Est-il éthiquement admissible qu'une circoncision en dehors de toute indication médicale soit pratiquée par un médecin et en milieu hospitalier? Est-il éthiquement admissible que cette intervention soit à charge de la sécurité sociale? Est-il éthiquement admissible que la loi traite différemment la circoncision masculine de la circoncision féminine?

Seuls deux points ont finalement fait l'objet de recommandations communes. Le premier est de suggérer d'abandonner le remboursement par la sécurité sociale, même lorsque la circoncision est pratiquée par un médecin: «Dans notre pays, une pratique semble conduire des médecins et des institutions de soins de santé à remplir, dans des documents relatifs aux soins de santé prodigués, les mentions nécessaires à ce que le coût d'une circoncision soit supporté, en tout ou en partie, par l'assurance-maladie. À cet égard, certains membres pensent que la circoncision n'est pas un soin de santé alors que d'autres la qualifient comme tel quand elle est le fait d'un médecin. Toutefois, tous les membres du Comité s'accordent pour affirmer que la charge financière de la circoncision non médicale ne doit pas incomber à l'ensemble des citoyens.»

Le second point de consensus met l'accent sur le recours à une pédagogie sociale suscitant "l'évolution des pratiques», ce qui suppose implicitement une interaction avec les acteurs collectifs aptes à réorienter ces pratiques, mais cette fois dans une perspective progressive d'autolimitation: "Le Comité propose, à l'unanimité, de réfléchir à surmonter les controverses en

39. Comité national belge de BioÉthiQue, Avis nº 70 du 8 mai 2017 relatif aux effets éthiques de la circoncision non médicale. 
encourageant l'évolution des pratiques vers la seule symbolique, de sorte que les rites continuent à se réaliser, mais sans inscription dans la chair de l'enfant. Ainsi, toutes les sensibilités religieuses seraient respectées sans qu'il soit porté atteinte à l'intégrité physique de quiconque.»

\subsection{LES POSITIONS DU CONSEIL DE L'ORDRE DES MÉDECINS SUR LES CERTIFICATS DE VIRGINITÉ}

En sa séance du 16 février $2019^{40}$, le Conseil national a examiné la problématique des tests et des certificats de virginité. Il estime que donner suite à une demande de rédaction d'une attestation de virginité pour raisons religieuses «n'a pas de justification». Pour le Conseil,

«le consentement et le respect de la patiente posent question. Ces examens sont souvent demandés par des tiers sans considération pour l'intimité personnelle et le droit à la vie privée de la personne concernée. Ils peuvent être vécus comme une agression. Ils entrânent une discrimination entre les femmes et les hommes dont les rapports sexuels échappent à toute évaluation de ce type. C'est un acte médical inutile pour la santé, sans pertinence scientifique et lourd de conséquences potentielles sur le bien-être de la patiente».

La conclusion demeure toutefois non impérative: «Le Conseil national de l'Ordre des médecins soutient la déclaration de l'OMS (2018) qui recommande aux professionnels de la santé de refuser de pratiquer ces tests et de délivrer des attestations de virginité.»

40. https://www.ordomedic.be/fr/avis/conseil/tests-et-certificats-de-virginite [consulté le 4 juin 2019]. 